\title{
Internet + VSDM and WebGIS System Design and Implementation of Corn Fertilizing Decisions
}

\author{
Yuxia Yao ${ }^{1, a}$, Guowei Wang ${ }^{2, b}$, Xiaoyan Liu ${ }^{1, c}$ \\ ${ }^{1}$ The Information Engineering College of Changchun University of Science and Technology, Ji Lin Chang \\ Chun 130600 China \\ ${ }^{2}$ The Information Technology College of Jilin Agricultural University, Ji Lin Chang Chun 130118 China \\ aemail:yx--yao@163.com; bemail: 41422306@qq.com; 'email: 932684698@qq.com
}

Keywords: WebGIS, spatial data mining, visualization, corn, intelligent decision making system

\begin{abstract}
In recent years as the rise of Internet + and geographic information system technology, the traditional GIS has a new development space, let a lot of further application of spatial data, decision making analysis on the space for us to provide the technical support. In the implementation of the national "863" project "corn precision operating systems research and application in the process, accumulated the massive spatial data on agriculture, to organize and manage these data, get the corresponding knowledge, better management, analysis and decision making, developed" WebGIS corn intelligent decision-making system based on Internet + "for application. The main contents include: using ArcIMS system to realize the basic operation of network geographic information system; Implements the new mode based on tiles splicing ways of network map browsing, when pan, in IE will have the effect of image stitching, shorten the waiting time for the user psychology, also to avoid the big picture of the large amount of data transmission; The visualization of spatial data mining results based on star coordinates is realized. The spatial data mining visualization of GIS is realized. The function of buffer analysis and variation chart is realized. The decision-making function of fertilization was realized. Combining analytic hierarchy process and fuzzy equivalent matrix method, spatial weighted fuzzy clustering algorithm is implemented.At the same time, the optimal classification number is obtained by using F distribution. By comparison, this method has higher precision and better effect than unweighted algorithm. In addition, this method is applied to the comprehensive evaluation of the difference of soil nutrient space, which is recognized and praised by experts of the review of agricultural engineering journal.
\end{abstract}

\section{Introduction}

Due to the spatial differences between corn fields is based on soil, soil nutrient spatial variation of the determination of precise fertilization, in order to ensure that farmers adjust measures to local conditions of fertilization, meet the need of national industrial structure adjustment of agriculture environment.The different space scale data, data in the different periods and different formats of data in a unified coordinate system to achieve efficient and orderly and the development of the centralized and unified management is the most effective way.Using digital technology to establish a new type of modern agricultural management mode, transforming the traditional agriculture to modern agriculture, promote the development of circular agriculture has very important practical significance ${ }^{[1]}$.Based on this idea, developed a fuzzy Data Mining based on the Visual space of the Internet + (Visual Spatial Data Mining, VSDM) and network geographic information system (WebGIS) of corn intelligent decision-making system is very necessary, the system could be used for agricultural production and management of various departments to provide decision-making reference information, provide users targeted scientific fertilizer and spraying guiding effect ${ }^{[2]}$.

\section{The overall design}

According to the structure of WebGIS and the demand of corn fertilizer decision system, the 
overall frame of the system is designed as shown in fig.1.

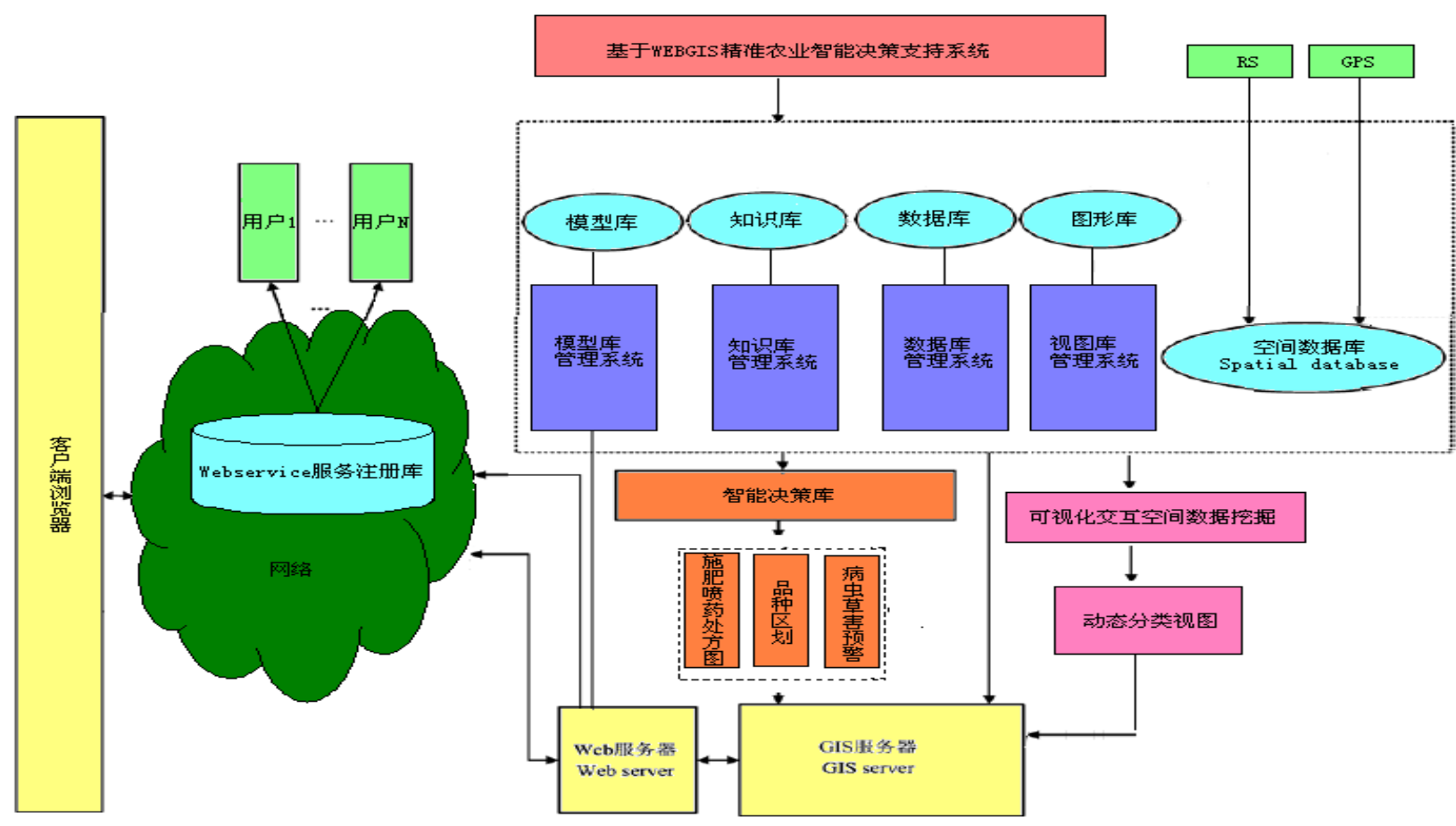

Fig 1 overall functional framework

\section{Database establishment}

(1) spatial database establishment: the spatial database is established mainly by two means: first, the paper makes use of the paper map to vectorization; The other is using GPS.

(2) paper map vectorization:

Computer configuration: hardware: CPU: PIII or higher, RAM: greater than 256M, HD: > 20G; Software: ArcView and ArcMap gis software for ESRI, USA.

Digital maps: first of all, the 1:50000 or 1:100000 paper map (such as soil map, administrative zoning map) scan, will scan in the online edition of TIF or JPG format, 24 true color, 300 dpi resolution. Second, the calibration of the electronic map in the ArcMap software, the paper (photo) on each major datum and the optional four (up, down, left, right), find the coordinates, if it is the earth coordinates (latitude and longitude) needs to be converted to the rectangular plane coordinates. The process is as follows:

Open ArcMap and select the location module Georeforencing, as shown in figure 2. Open to locate scan map file in Georeforencing option buttons on the toolbar, find the coordinates of known points on the graph, the right name dialog box, enter the rectangular plane coordinates ( $\mathrm{X}$ to 8 digits, $\mathrm{Y}$ for seven figures, the coordinates on the graph of insufficient use 0 is lacking, as shown in figure 3), latitude and longitude position need to generate a standard grid (as shown in figure 4), calibration, repeatedly until the error is close to zero.

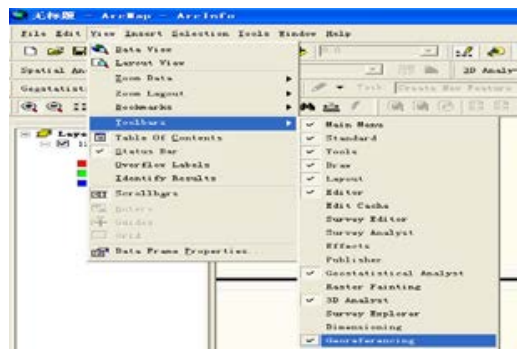

Fig2Positioning module selection; Fig3Enter Registration coordinates;

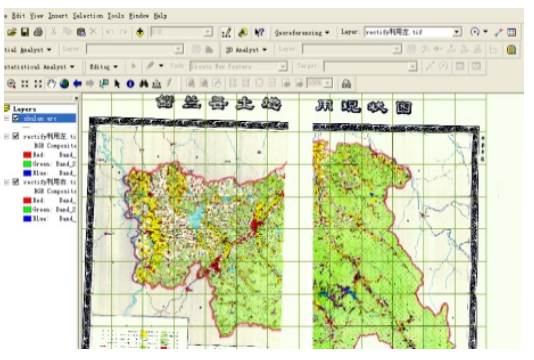

Fig4 Standard grid generation

Regenerates a grid file after the location of the file output. Select the Rectify in the 
georeforexample menu... Options (as shown in figure 5), enter the grid size, type and name in the pop-up dialog box (see figure 6), and click the OK button to store it.

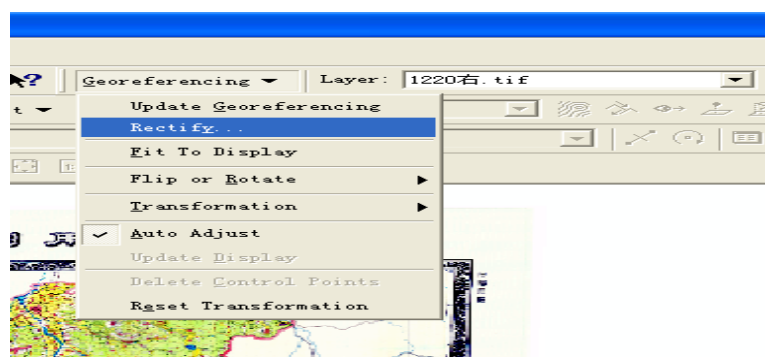

Fig5 Rectify ... Options

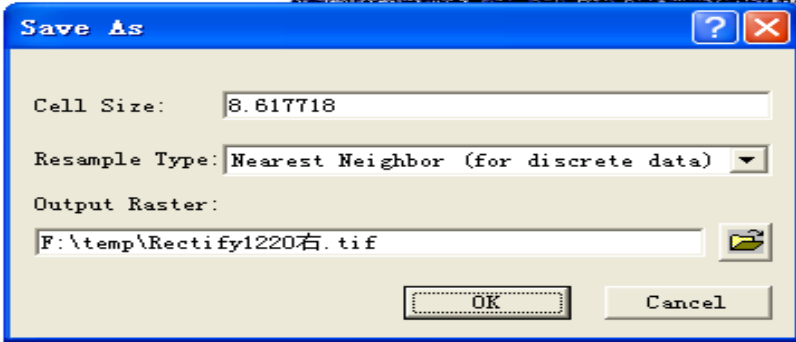

Fig6 Export Raster File dialog box

Finally, the map vector is vectorized with ArcView software. The process is to open the calibrated map in the ArcView and create a new file (point, line, or face file, whose properties and layout are table 1). Use the tool shown in figure 7 to vectorize the graph. Note: the boundary (outer outline) is usually drawn first when the document is drawn. Then the boundary (outer contour polygon) is cut with the drawing of the polygonal cutting tool. After the drawing of the independent polygon is finished, we need to separate the two tabula with Subtract Features. ${ }^{[3]}$

Table1 New File Types

\begin{tabular}{|l|l|l|}
\hline Point files & $\begin{array}{l}\text { Attribute } \\
\text { are defined in data tables such as: residential, } \\
\text { elevation, text, etc. } \\
\text { are defined in data tables such as roads, } \\
\text { railways, linear rivers, etc } \\
\text { defines the fields for each specialized element } \\
\text { in the data table. }\end{array}$ & $\begin{array}{l}\text { byow different definitions by the thickness, } \\
\text { shape and color of the line. } \\
\text { The shape and color of the soil map, the surface } \\
\text { of the river and other elements }\end{array}$ \\
\hline & & \\
\hline
\end{tabular}

After the vectorization, you need to add a property value to it, as shown in figure 7 and figure 8 .

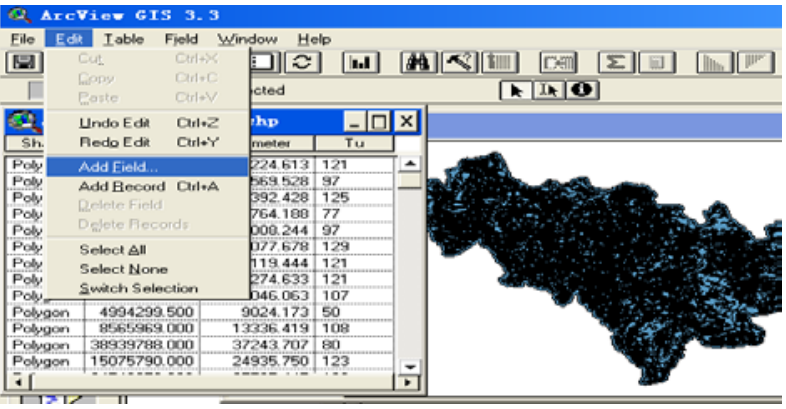

Fig7 Add a field option

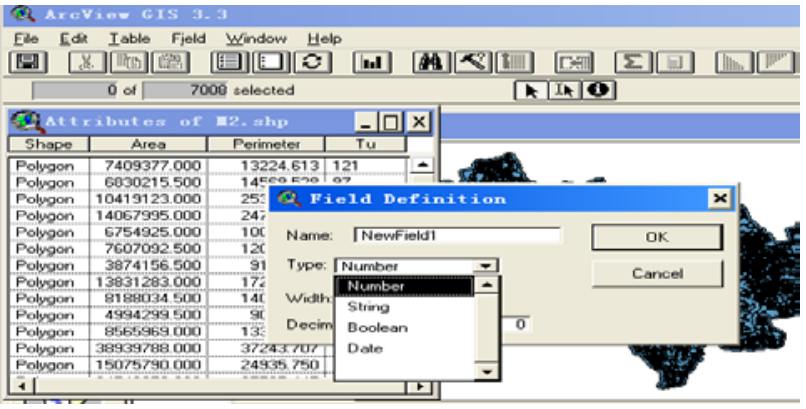

Fig 8 Setting the field names and types

Vectoring result:Vectorization maps of the arbor town, nongan county and lishu county were established using vectoring method (see Fig. 9, Fig. 10, Fig. 11).
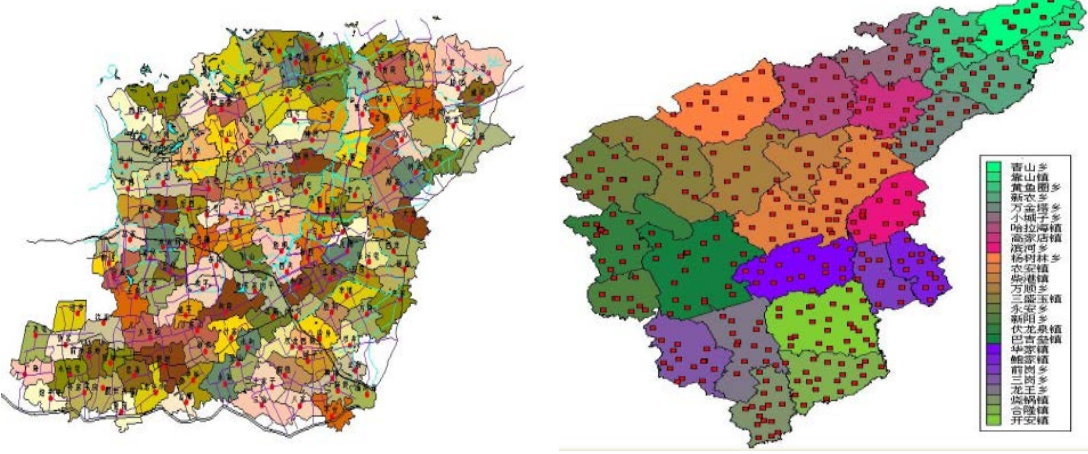

Fig 9 Vector Map of Yushu City gongpengzi; Fig 10 vector map of Nongan ;

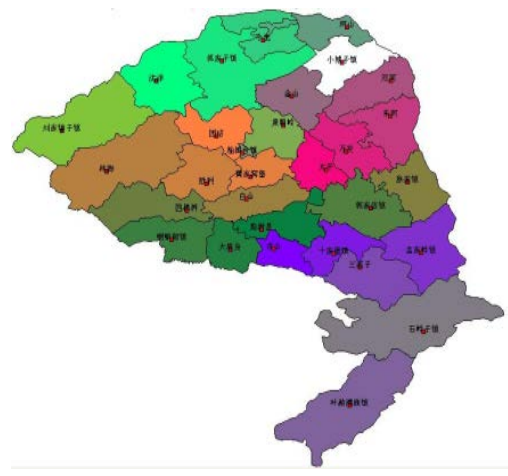

Fig 11 vector map of Lishu 


\section{(3) GPS collection}

The GPS device USES the American Trimble's AgGPS differential GPS station and a set of receivers to achieve the accuracy of the sub-meters.Data acquisition software acquisition process in U.S. DeltaDataSystem company's Pocket DLog software, the software in the connection of handheld computer graphics equipment and global positioning system (GPS), can provide abundant data acquisition function ${ }^{[4]}$. The process of collecting data is as follows:

Run the Pocket DLog software, click the File menu, select the GPS option under Properties (see figure 12), and you can pop up the interface shown in figure 12.

In the interface shown in figure 12, the Port option from COM1 to COM8 can be selected according to the actual connection Port, and the default is COM1; The Baud option has 2400, 4800, $9600,14400,19200,38400,57600$ and $115 \mathrm{~K}$. The setting is the same as that of the Baud in the GPS receiver, which normally USES the 9600 band. The Type option has three options, NMEA, StarFireRTK and RTK NMEA, which are usually set to NMEA. The Icon option is the display Icon for the GPS location at the sampling time; Set the GPS option to On; To ensure that the sampling accuracy is within one meter, the DGPS option should be set to On. After all options are set, click the OK button.

After setting the GPS option, click on the New Project under the File menu... Option, click OK button to confirm, enter new project setting interface. Enter the name of the new project, click the OK button to create a new project.

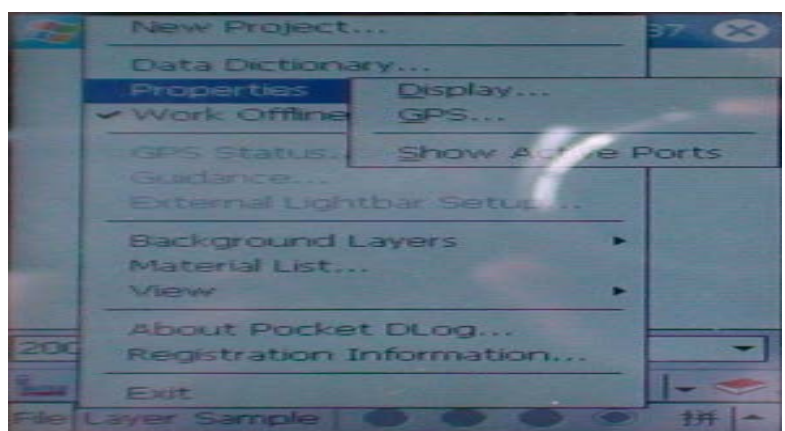

Fig 12 GPS option

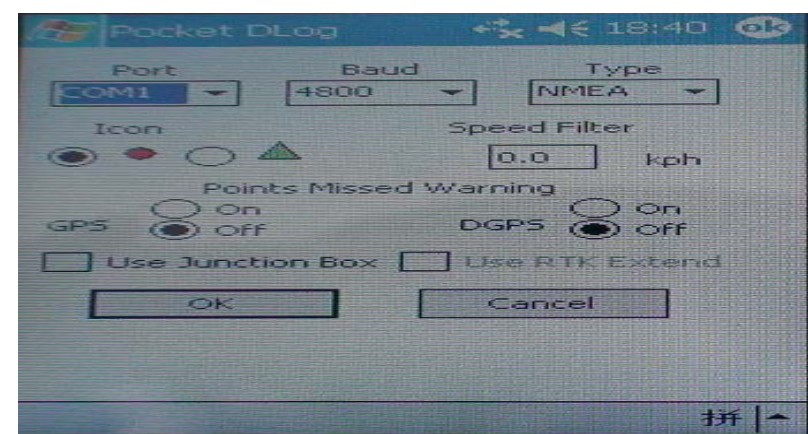

Fig 13 GPS settings

Click New under the Layer menu. Option, pop up the dialog shown in figure 17, and enter the Name of the created Layer from the Layer Name option in the dialog box. If you don't select the No Attribute option, you need to set the Attribute name, type, size, and precision of the layer to create multiple properties. Otherwise, no attributes are added. In the Object Type option, select the layer Type, which is mainly point Type, line Type, and surface Type. Set the layer to display the color ${ }^{[5] .}$ Click OK button to sample, and figure 18 is the sampling schematic.

After the sample is completed, connect the PDA and microcomputer, and use Microsoft's AciveSync simulator to keep the PDA and microcomputer in sync. Using the Pocket Convert software, the collected data can be converted into AutoCad DXF, ESRI shape, MapInfo MIF, XYZ Text and Soil Lab DBF files. The data in this paper are all shape files. To run ArcInfo software, open the transformed data, grid the data of the collected land, and get the sampling grid, the grid map of the block town and 8 town of yulin, jilin province.

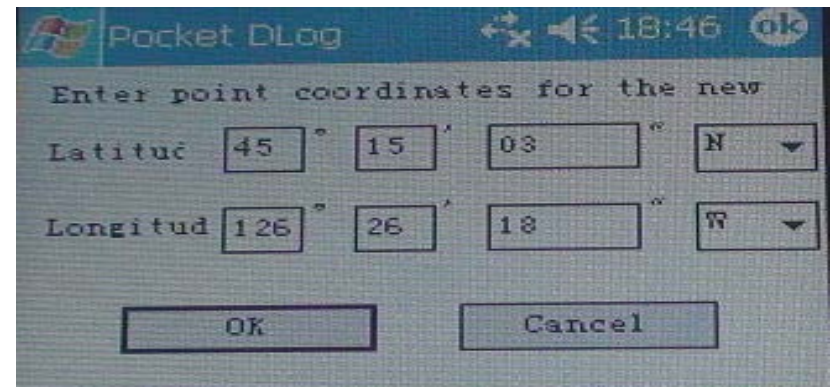

Fig 14 set coordinates

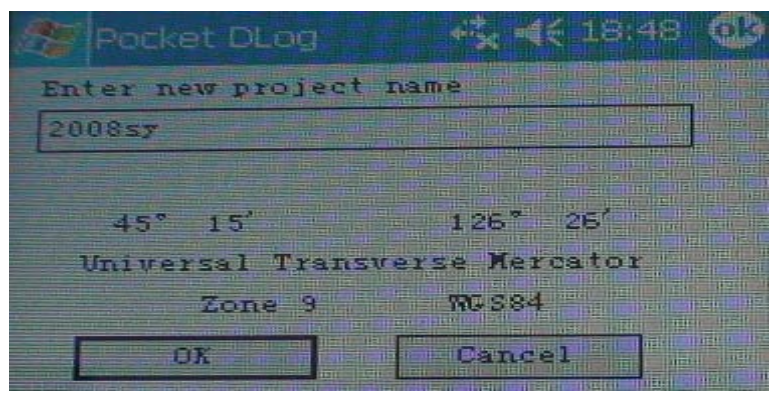

Fig 15 Create a new project 


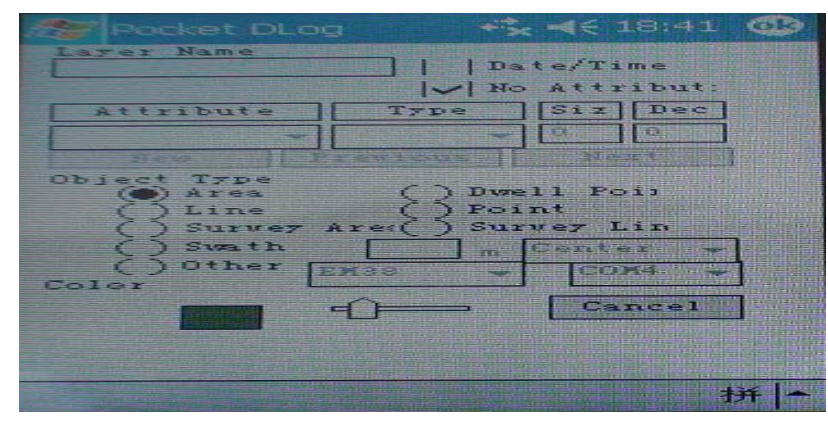

Fig 16 Create a new layer

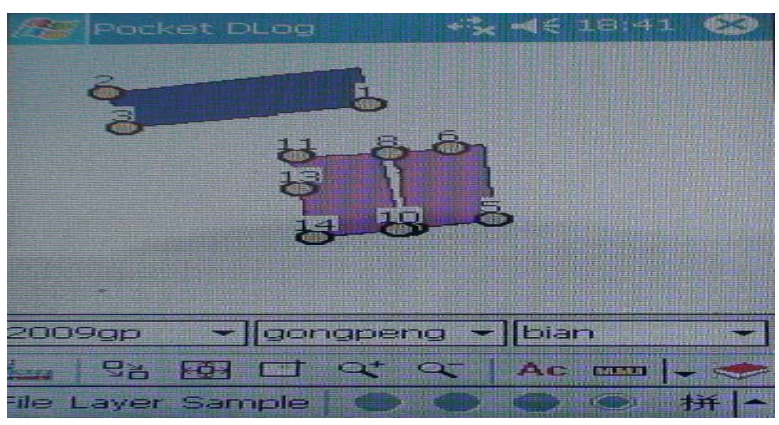

Fig 17 Schematic diagram of sampling

\section{Attribute database setup}

According to the sampling grid map, soil samples are taken at the corresponding sampling grid points, and the data is saved to in the property database. ${ }^{[6]}$

(1) operation function implementation

The Toolbar (Toolbar) most browsers support 11 kinds of roaming and scaling tools, except that the HTML browser does not support Next Extend. Toolbar implements zooming and roaming functions for basic map operations.

Measurement tools are a quick way to Measure the length of a line between two points. As with the buffer tool, the measurement tool also requires the default map unit to be defined in the Author or by the user using the Set Units tool ${ }^{[7]}$. When is used for measuring tools, the length of segment length and its total length will be shown simultaneously. Use the Clear Selections tool can remove when measuring the painting line, but change the scope of view does not make them disappear.

There are many options for selecting elements in ArcIMS for Graphical selection tools, such as Query Builder, Find Tool, Search Tool, or buffer Tool. In addition, elements can be selected by drawing a rectangle, circle, or multilateral line on the graph, and then they are intersected by the graph. After the elements are selected, they become part of the selection set, whose property values can be listed in table form, or buffer analysis using buffer tools. Clear All Selections tool to remove All of the selection. Because the execution of the graphical selection tool varies greatly from client to client, this is a great tool for testing which templates to use when creating a site.

Finding (Find) lookup tools is another way to query spatial databases. The Find tool is a commonly used lookup tool that does not require an active layer. In the Java browser, the lookup tool can query all of the fields of the layer, and the query is case sensitive. In the HTML browser,

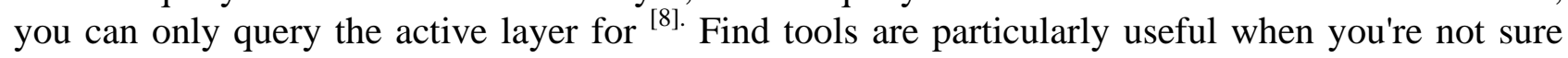
that the table is all open or that the user is not familiar with the database operation. At the same time, the user can select the selected set to return and roam or scale to the highly selected record.

Another way to Query a spatial database is to Query for SQL queries by predefined queries. Predefined queries are defined in Author by a logical expression with a variable. On the client side, the user submits only the value of a variable to the system, which can be queried. Similar to Query Builder, users can choose not to download all records if the Query result exceeds 100 records. Similarly, you can select a record from the list and scale to the corresponding view to display ${ }^{[9]}$. Although predefined queries limit the ability of users to build complex queries, they can guarantee that the query will not fail because it is previously detected. If the user wants more flexible queries, use the Query Builder tool.

The Query Builder Query queries the spatial database and returns the Query results in tabular form. The user can create a logical expression through the input or selection method, and the expression is committed to the server when the Excute is pressed. If the returned result exceeds 100 records, the user can choose not to download the selection set for all records. This option may fail when the query results exceed the expected elements. It is sometimes difficult to understand this phenomenon. When all records are not downloaded, some functions in the menu such as highlighting, roaming, zooming, statistics, etc., may fail. 
The basic statistical functions in the query include the average value, maximum, minimum value, standard variance, and sum of all elements or elements. Similarly, the query can be used to map the range of maps that are highlighted by highlighting elements.

Buffer (Buffering feature) buffer analysis is a very important analysis function of GIS. In ArcIMS, the interface of the element's buffer analysis is very simple. The user simply selects an active layer and USES the query tool to create a select set for the buffer analysis. With the selection set, you can use the buffering tool. Enter a buffer distance and select a buffer unit. If it is a Java browser, users can also select the elements within the buffer within another topic. This process is a little like the choice of the topic in ArcView. Note that when a buffer is analyzed, anything that is connected to any part of the buffer becomes part of the selection set.

(2) ArcIMS implement tile splice technique

Before Google Maps, all kinds of network map in the technology with the method of traditional WebGIS, using Java Applet, SVG, dynamically generated map image, and Google Maps USES Ajax and tiles splicing scheme to build the new mode of network map has been widely recognized.

To build a Google Maps style web map, you first need to have a Google map-like JS library as a client. The second is how to connect the client to ArcIMS, how to cache images, and so on ${ }^{[10]}$.

In the HTML file, the JS code for creating a map is as follows:

function IMSMapType() \{

MapType.apply(this);

this.getSrc $=$ function(level, row, column $)\{$

return "arcims.asp?z="+level+"\&x="+column+"\&y="+row

\} \}

MapModel.mapTypes = new Array(new IMSMapType());

MapModel.maxZoomLevel=10

This code creates a map type, you can also create a variety of types of Maps (similar to Google Maps), including getSrc function defines how to return the picture method, there are three parameters: scaling, horizontal position and vertical position ${ }^{[11] .}$ In the library of MapEasy, images of different scales are spliced in the same subscript as in a two-dimensional array.

In arcims. Asp, the main program flow shown in figure 18, first of all, according to the location of the image parameters, constructing images, and then judge whether there is, if it exists, is returned, otherwise call arcims rendering and save the image. The return images do not use redirection to the image, but in the way they write the data stream.

(3) implementation of fertilization decision

Assumptions:

Sf: fertilizing amount; Cl: corn target yield; Xs: the absorption component of corn grain for every $100 \mathrm{~kg}$ of corn; CD: soil nutrient determination value;

Ys: effective nutrient conversion coefficient of soil; Hl: nutrient content of fertilizer; Ly: fertilizer rate utilization rate, nutrient balance method fertilization model is:

$$
s f=\frac{c l \times x s-c d \times y s}{h l \times l y}
$$

The mathematical model of fertilization of phosphate fertilizer in nongan county was calculated as an example (the content of P2O5 in the diammonium of diammonium was $46 \%$

Corn target yield*0.007-0.3* Soil nutrient content*Soil effective nutrient conversion coefficient

Fertilization of phosphate fertilizer=

\subsection{6*Fertilizer efficiency}

(4) implementation of spraying decision-making function

Assumptions:To indicate the grade of weed density; Indicates the density of weed, unit is strain/meter 2; The dosage was diluted 200 times.According to the classification of weed density, the formula is as follows: 


$$
d j=\left\{\begin{array}{l}
1, m d \in(0,30] \\
2, m d \in(30,70] \\
3, m d>70
\end{array}\right.
$$

Different weed density levels were applied to the corresponding dosage, and the model was as follows:

$$
y l=\left\{\begin{array}{l}
40 \mathrm{~kg}, d j=1 \\
50 \mathrm{~kg}, d j=2 \\
60 \mathrm{~kg}, d j=3
\end{array}\right.
$$

(5) spatial fuzzy algorithm implementation

The fuzzy equivalence matrix is based on the fuzzy equivalent matrix clustering algorithm, which USES the detailed design tool - problem analysis diagram (PAD) to describe the algorithm. FCM algorithm implementation is based on the principle of FCM algorithm, which USES the detailed design tool - problem analysis diagram (PAD) to describe its algorithm. The analytic hierarchy process (ahp) is based on the principle of analytic hierarchy process (ahp), which USES the detailed design tool, problem analysis diagram (PAD), to describe the algorithm of ${ }^{[12]}$.

\section{Conclusion}

Introduces the WebGIS system in this paper, spatial data mining and visualization of data mining method, the integrated application of WebGIS, expert system, decision support system and visual space fuzzy data mining technology, developed a "VSDM and WebGIS based intelligent decision-making system of corn". The main innovation points have the following aspects:

(1) the WebGIS, expert system, decision support system and visual spatial fuzzy data mining technology is applied to the system implementation, so as to achieve the management of spatial data, analysis, and decision making.

(2) a spatial weighted fuzzy dynamic clustering algorithm is proposed based on the hierarchical analysis method. The results show that the classification results of the algorithm can be more objective and accurate. Main performance:The algorithm can distinguish noise effectively. The algorithm can fully consider the imbalance between each property.

By introducing F - distribution in the algorithm, the optimal threshold value is obtained and the optimal threshold is obtained by using the optimal threshold of the optimal classification.

(3) the 2 mentioned in algorithm applied in the variable fertilization to improve the comprehensive evaluation on the spatial heterogeneity of soil nutrients, has been in the same plot after years of continuous variable fertilizer can improve the spatial heterogeneity of soil nutrients to the conclusion that the "journal of agricultural engineering review experts, evaluation for" on the thinking method has certain innovation value, the overall high academic value and research significance ".

(4) combining entropy weight method in information theory, this paper proposes a new algorithm for FCM algorithm based on entropy weight method. The entropy weight method is the difference between the data itself and the actual distribution of data. In addition, entropy weight method does not need to score and input data, which reduces the influence of subjective factors, and has better automation and intelligence. The results are higher than the unweighted FCM algorithm by analyzing both the Iris and Wine data sets.

(5) realize the visualization of spatial data mining results in two ways: one is realized by using star coordinates; The other is based on GIS. Visual results can help us to analyze and make decisions about data.

(6) according to the nutrient content of the community, the fertilization decision was realized, and the fertilization rate of each community was obtained. Based on this, the application of fertilization recipe is generated to provide the data basis for the operation of the variable fertilizer machine.

Currently, the function of the theoretical research has been completed, the algorithm of each part 
is programming implementation, and as long as a little modification, the algorithm can be applied to other areas, the portability is good. However, the weighted FCM algorithm has not been integrated into the system yet.

\section{References}

[1] Chen yunping, Zhao chunjiang et al. Based on knowledge model and the precision agricultural prescription intelligent generation system of WebGIS [J], China agricultural science, 2007, 40 (6) : 1190-1197

[2] Chen guifen, wang yue, The research and application of the accurate fertilization system of maize [J], journal of jilin agricultural university, 2006:586-590

[3] Zhu bonyao, analysis and design of agricultural industrialization information system based on WebGIS [J]. Agricultural systems science and comprehensive research. 2007, 23 (2) : 217-219

[4] Yao yuxia, based on the research and application of the expert system for the diagnosis and treatment of cordyceps in jilin corn, [J] jilin agriculture, 2014 (10) 102-103.

[5] Yuxi-Yao, The Research and Application of Remote Sensing Technology in MaizeYield Estimation of Jilin, [J] ieee

[6] li yajun,Research and practice of the diagnostic system of corn disease, [J] agricultural disaster research, 2012 (5) 129-130.

[7] Chen guifen The application of Fuzzy cluster analysis of in soil nutrient classification [J], corn science, 2008, 16 (2) : 118-121

[8] $\mathrm{Wu}$ yuhua, ye ping. Analysis of restriction factors for the promotion of "intelligent decision-making system for corn production management" [J]. China agriculture bulletin. 2009, 25 (10) : 258-263

[9] Chiang wave, zhu qiang, qian xudong. Development practice manual of WebGIS - based on ArcIMS, OGC and tile GIS [M]. Electronic industry press

[10] Sun JG, Liu J, Zhao LY. Clustering algorithms research [J]. Journal of Software, 2008, 19 (1) : 48-61.

[11] Jia zeyu, liu yaolin. Research on visualization spatial data mining [J]. Geospatial information. 2009, 7 (6) : 10-15

[12] Yao yuxia, research and practice of corn yield prediction based on remote sensing technology, heilongjiang technical information, 2013(2)29-30 